\title{
La colaboración entre estudiantes universitarios y negocios sociales
}

\author{
Germán Osorio-Novela, Alejandro Mungaray-Lagarda y Natanael Ramírez-Angulo
}

\section{RESUMEN}

Este documento se enmarca en los modelos pedagógicos de investigación-acción y aprendizaje-servicio, a través de la operación de un programa de asistencia a negocios sociales. Se comparte la operación de un programa de investigación, asistencia y enseñanza a empresas, en donde estudiantes de último grado de licenciatura de la Universidad Autónoma de Baja California, México, actúan como consultores de micronegocios emprendidos en zonas de alta marginación, asistiéndolos en temas de desarrollo empresarial y personal. De esta forma, de 1999 a 2019, han participado más de 1720 estudiantes en 12790 negocios sociales, impactando en su fortalecimiento como fuente de ingresos de familias en condiciones de pobreza. Los hallazgos arrojan resultados deseables en los mecanismos de aprendizaje para estudiantes, a través de experiencias reales con empresas en contextos de alta marginación, mejorando su sentido solidario, emprendedor y de colaboración.

Palabras clave: proceso enseñanza-aprendizaje, necesidades sociales, programas de entrenamiento, México.

\section{Germán Osorio-Novela}

gosorio@uabc.edu.mx Mexicano. Doctor en Ciencias Económicas, Universidad Autónoma de Baja California (UABC), México. Profesor-Investigador, Facultad de Economía y Relaciones Internacionales, UABC, México. Temas de investigación: economía de la micro y pequeña empresa, vinculación universidad-negocios sociales. ORCID: https://orcid.org/0000-0003-0157-6979.

\section{Alejandro Mungaray-Lagarda}




\title{
A colaboração entre estudantes universitários e negócios sociais
}

\section{RESUMO}

Este documento se enquadra nos modelos pedagógicos de pesquisa-ação e aprendizagem-serviço, através da operação de um programa de assistência a negócios sociais. É oferecido a operação de um programa de pesquisa, assistência e ensino a empresas, onde estudantes de último grau de licenciatura da Universidad Autónoma de Baja California, México, atuam como consultores de micro negócios empreendidos em zonas de alta marginalidade, assistindo-os em temas de desenvolvimento empresarial e pessoal. Desta forma, de 1999 a 2019, têm participado mais de 1720 estudantes em 12790 negócios sociais, impactando em seu fortalecimento como fonte de renda de famílias em condições de pobreza. Os resultados mostram beneficios desejáveis nos mecanismos de aprendizagem para estudantes, através de experiências reais com empresas em contextos de alta marginalidade, melhorando seu sentido solidário, empreendedor e de colaboração.

Palavras chave: processo ensino-aprendizagem, necessidades sociais, programas de treinamento, México.

\section{Collaboration between university students and social entreprises}

\begin{abstract}
This paper is framed in the pedagogical models of action research and service-learning, through the operation of a program of assistance to social entreprises. It shares the operation of a program of research, assistance and teaching to businesses in which undergraduate students of the Autonomous University of Baja California (Universidad Autónoma de Baja California, Mexico) act as consultants to micro-entreprises in highly marginalized areas, assisting them in business and personal development issues. In this way, from 1999 to 2019, more than 1,720 students have been engaged in 12,790 social businesses, impacting their strengthening as a source of income for families living in poverty. The findings yield desirable results in learning mechanisms for students, through real experiences with businesses in highly marginalized contexts, improving their sense of solidarity, entrepreneurship and collaboration.
\end{abstract}

Key words: teaching-learning processes, social needs, training programs, Mexico. 


\section{Introducción}

El programa de investigación, asistencia y enseñanza a negocios sociales, inició en su fundamento básico en 1999, como un mecanismo práctico de enseñanza universitaria asociado principalmente a los conceptos de investigación-acción y aprendizajeservicios. Es un programa que expone un método de aprendizaje que vincula los temas vistos en clases con algún contexto local, a través de servicios voluntarios de estudiantes en la comunidad. La intención pedagógica principal es que los alumnos sean capaces de relacionar las experiencias prácticas de una empresa con el conocimiento educativo adquirido en su licenciatura, fortaleciendo su responsabilidad social. Para ello, se busca que los estudiantes aprendan y experimenten actividades emprendedoras con base en consultorías a dueños de microempresas sociales surgidos de población marginada, en donde el autoempleo ha sido la alternativa principal de ingreso y sustento para dicho sector.

El programa de investigación, asistencia y enseñanza a negocios sociales se ha complementado con mecanismos innovadores de educación, que reestructuran los procesos de aprendizaje dentro del aula, analizando escenarios y problemáticas reales de empresas locales, desarrollando habilidades útiles de aplicación de conocimiento y experimentando acciones emprendedoras. El objeto de este programa se ha centrado en la reestructuración del proceso de aprendizaje destinado a fomentar la educación, cultura empresarial y acciones de creatividad en los estudiantes, mediante la promoción en la creación y soporte de empresas con responsabilidad social (Barba y Atienza, 2016).

Desde una perspectiva innovadora de colaboración conjunta, este documento también describe los elementos y circunstancias en los cuales este programa pedagógico transitó a un programa público para el desarrollo microempresarial y el empleo, en colaboración con el gobierno del estado de Baja California, México, y la Universidad Autónoma de
Baja California (UABC), en donde los estudiantes y microempresarios sociales fueron los actores principales. Además, se expone la labor integral que el programa ha desempeñado desde su inicio en 1999 hasta 2019, con la colaboración de más de 1720 estudiantes universitarios y 12790 micronegocios.

En el segundo apartado de este documento se detalla un marco teórico básico respecto a temas pedagógicos de enseñanza y aprendizaje, a través de los conceptos y dimensiones actuales de los métodos de investigación-acción y aprendizaje-servicio. En el tercer apartado se expone el contexto social y empresarial observado en México en los últimos años, y el papel que ha desempeñado el gobierno y las universidades en temas de responsabilidad social. En el cuarto apartado se exponen los conceptos asociados a emprendimientos sociales y negocios sociales, como un método alterno y sostenible de intervención social. En el quinto apartado se describen los antecedentes e inicios de este programa de aprendizaje-servicio de la UABC. En el sexto apartado se explica la metodología de este programa, en función del papel protagónico y responsable de estudiantes universitarios como consultores para el desarrollo de negocios sociales. El séptimo apartado expone los resultados alcanzados en materia educativa y formativa para los estudiantes, y también en fortalecimiento del sector empresarial social. Finalmente, en el apartado de conclusiones se presentan los alcances del programa y los retos para el seguimiento de proyectos que impulsen el aprendizaje en comunidad, mecanismos innovadores de enseñanza, el sentido solidario, emprendedor y de colaboración social.

\section{Fundamentos teóricos básicos de los conceptos de investigación-acción y aprendizaje-servicio}

Si bien el concepto de investigación-acción, definido como aquella investigación realizada por profesores dentro de la misma actividad docente con el propósito de visualizar e implementar áreas de mejora en 
los procesos de enseñanza (Bausela, 2004), tiene su inicio desde la década de 1940, actualmente existen desarrollos y programas pedagógicos que mantienen su vigencia, con mecanismos y adiciones innovadoras, reconociendo su utilidad en incrementar y optimizar el proceso de aprendizaje en los actores participantes.

Se ha fundamentado que la implementación eficaz del método de investigación-acción presenta su propio dinamismo de actualización operativa, valorando una naturaleza cíclica en su metodología. Esto se logra gracias a que las conclusiones de la investigación siempre deben converger en la resolución del problema expuesto inicialmente, poniendo en práctica la premisa de que la investigación-acción no es un proceso de análisis sobre la acción, sino un aprendizaje en la acción. Con ello, este método ofrece un mecanismo en donde el profesor dimensiona el efecto de sus acciones mientras son implementadas. De tal forma que la importancia de este paradigma no radica en su sentido científico, sino en su función cualitativa para incrementar la labor docente (Cabrera, 2017).

En la educación profesional, la evidencia empírica ha subrayado la importancia de la investigación-acción como una técnica fundamental para incrementar el desempeño profesional de los docentes, al generar conocimiento innovador a través de la investigación. La combinación entre la teoría y práctica parece un factor importante para que se puedan encontrar las soluciones a las posibles dificultades encontradas en el proceso investigativo, mediante la propia acción de los actores involucrados (Pérez-Van-Leenden, 2019).

Por ello, en los últimos años se ha puesto sobre la mesa la pertinencia de asociar la investigaciónacción al dinamismo generado del Aprendizaje Basado en Proyectos (ABP), consideradas dos metodologías complementarias, en el sentido que la primera se desarrolla en el contexto de la investigación, mientras que el segundo lo hace en el proceso didáctico-práctico. En ambas metodologías se resalta la necesidad de generar procesos cíclicos de aprendizaje, en donde se fortalece la actividad docente mediante la participación y retroalimentación activa de sus actores (Botella y Ramos, 2019).

El Aprendizaje Basado en Proyectos es una herramienta cada vez más utilizada en los procesos pedagógicos y docentes, sobre todo en planes y propósitos con fines solidarios y comunitarios; estos conceptos incluso han sido la base de proyectos macros, por ejemplo, los llamados SmartCities y Ciudades Educadoras, en donde se valora no sólo el proceso eficiente de aprendizaje, sino la formación sólida de personas con valores, solidarias, con comportamiento ético y alto sentido de cohesión social. Así, literatura reciente ha señalado que las metodologías de investigación-acción y de ABP, comparten tres factores indisolubles: la necesidad, el aprendizaje y el servicio (García et al., 2018).

Con base en la combinación de estos elementos, se establece el concepto de aprendizaje-servicio, a través de una metodología netamente pedagógica con la capacidad de asociar y cumplir cabalmente la formación de personas con sentido social y ciudadano (Ruiz-Corbella y García-Gutiérrez, 2019). Este es un objetivo que debe priorizarse en las instituciones de educación, con el fin de desarrollar ciudadanos con valores sociales y participativos, contextualizados en la comunidad local.

El proceso de aprendizaje-servicio se ha reflejado, por ejemplo, en las instituciones de educación superior, a través de la prestación de servicio social o comunitario, logrando que este dinamismo combine la comprensión de contenidos curriculares con un servicio práctico a la sociedad, en donde se resalte la adquisición de competencias técnicas y solidarias, alcanzando una metodología de aprendizaje a través de la comprensión de contextos reales, de tal forma que los estudiantes tengan la oportunidad de ser un actor principal en el ofrecimiento de un servicio profesional a la comunidad (Chiva et al., 2018). 
Diversas universidades alrededor del mundo han implementado esta filosofía de aprendizaje-servicio, al reconocerlo como un proceso en donde pueden conjuntarse visiones de educación basada en competencias. Cuando se implementa un mecanismo de servicio solidario, en donde el estudiante debe atender necesidades genuinas y reales de su comunidad, brinda la oportunidad de contrastar la verdadera adquisición de sus competencias, desarrollando una visión estratégica, no con base en supuestos imaginarios, sino con situaciones reales, incrementando el sentido competitivo del estudiante mediante una planeación de contenidos formativos y curriculares de su programa educativo.

Se ha establecido, a su vez, que la clave de esta metodología para una formación integral, es permitir que el estudiante contemple todos los componentes principales de un servicio comunitario profesional: estudio del contexto local, creación, planeación, desarrollo, implementación y evaluación del proyecto (Mendia, 2016). De esta forma se brinda el sentido cíclico de aprendizaje y mejoría para futuras aplicaciones.

Algunos de los retos y factores determinantes que se deben considerar al momento de poner en práctica el método de aprendizaje-servicio, son: adquirir nuevo conocimiento y desarrollo personal; participación activa y sistemática; alto nivel de organización; considerar las necesidades de la comunidad; coordinación entre la institución educativa y la comunidad receptora del servicio; alto nivel de responsabilidad cívica; reflexión profunda de la experiencia vivida (Rubio y Escofet, 2018).

\section{El contexto social y empresarial en México, perspectivas desde la responsabilidad universitaria}

El Consejo Nacional de Evaluación de la Política de Desarrollo Social (CONEVAL) es la institución pública-autónoma encargada de medir la pobreza en México, y evaluar el impacto que los programas y políticas sociales tienen en la reducción de ésta. La definición de pobreza que considera este organismo es multidimensional. A nivel internacional sólo suele considerarse el estándar de ingreso para determinar si una persona se encuentra en condición de pobreza. La medición oficial que se realiza en México, además del ingreso, considera el acceso o carencia de los que se llaman derechos sociales, como el derecho a la educación, a los servicios de salud, a la seguridad social, a la calidad y espacios de la vivienda, a los servicios básicos en la vivienda, a la alimentación y el grado de cohesión social (CONEVAL, 2017).

Los resultados de la última actualización muestran que, a nivel nacional, el porcentaje de población en condición de pobreza es de $43.6 \%$, reflejando una disminución con respecto al $46.2 \%$ del 2014. En términos absolutos hay en México alrededor de 53.4 millones de personas en condiciones de pobreza multidimensional. Por una parte, la pobreza extrema - un subgrupo con mayor vulnerabilidad, pasó de $9.5 \%$ a $7.6 \%$, que en términos absolutos significan 9.4 millones de mexicanos-, considerada así cuando las personas tienen más de tres carencias sociales de las seis posibles, y que además se encuentran por debajo de la línea de bienestar mínimo; es decir, de un ingreso monetario tan bajo, que si se dedicara completo a la adquisición de alimentos, no podría adquirir los nutrientes necesarios para lograr una vida sana (CONEVAL, 2017).

Particularmente, en el estado de Baja California, el porcentaje de población en situación de pobreza es de $22.2 \%$, que representa un avance en la región con respecto al 28.6\% registrado en 2014. En términos absolutos significan 789 mil personas en condiciones de pobreza; 40 mil de las cuales $-1.1 \%$ de la población estatal - se encuentran en pobreza extrema (CONEVAL, 2017).

Indicadores de pobreza del país por grupos de edad, permiten visualizar que después de los indígenas, los menores de 18 años se encuentran en situación de pobreza extrema de manera importante 
(28\% y $9 \%$, respectivamente) y de pobreza moderada $(43.8 \%$ y $42.1 \%$, respectivamente). Esto muestra que el $51.1 \%$ de los menores de edad en México se encuentran en pobreza, lo que provoca la reflexión sobre el futuro que les espera durante su etapa de adultos, con actividades asociadas a la formación de su familia y a su quehacer productivo-laboral.

Ante este escenario adverso, el emprendimiento de un negocio social, en la figura de autoempleo, parece ser una solución alterna y viable a los problemas de ingreso, consiguiendo de esta manera satisfacer las necesidades básicas de los emprendedores y de quienes de ellos dependen (Premchander, 2003; Ramírez et al., 2017). Las condiciones dispares bajo las cuales se desenvuelve el segmento empresarial, ha dado como resultado un gran número de unidades económicas de tamaño micro, que operan bajo condiciones de ineficiencia económica, pero que se han convertido en una opción de desarrollo para aquellas familias que se encuentran en condiciones de pobreza. Esta afirmación coincide con Yunus (2013) y Premchander (2003), quienes consideran al sector microempresarial y su desarrollo como un mecanismo para reducir los niveles de vulnerabilidad social, dada la capacidad que tienen estas unidades productivas de ser generadoras de empleo, ingreso y coadyuvar en la recuperación del bienestar en momentos de tensión social y crisis económica (Kantis et al., 2004; González et al., 2018).

Una problemática vinculada a la pobreza está asociada a deficiencia en capacidades productivas y empresariales por parte de jóvenes que viven en zonas de marginación (Barbero y Cortés, 2005; PNUD, 2016).

En México, según el Consejo Nacional de Población (CONAPO), en 2017 la proporción de jóvenes entre 10 y 24 años es de $27.8 \%$, cuyas características educativas reflejan grandes desafíos por resolver, ya que en 2015 sólo el 67.5\% de los jóvenes entre 15 y 19 años contaba con secundaria terminada, $41.8 \%$ con al menos un año de educación media superior y sólo el 4.1\% había logrado ingresar a estudios de educación superior.

De acuerdo con el informe "Perspectivas económicas de América Latina: juventud, competencias y emprendimiento, 2017" de la Organización para la Cooperación y el Desarrollo Económicos (OCDE), invertir en competencias de los jóvenes es esencial para poner en funcionamiento fuentes endógenas de desarrollo y construir una base sólida para el progreso futuro. Entre las sugerencias principales está el impulso al emprendimiento social, como herramienta que facilite el salto de la escuela al trabajo, a partir de la creación de ecosistemas de emprendimiento para emprendedores de alto crecimiento y promotores de la movilidad social (OCDE, 2017).

En un estudio publicado en 2018 por el Acreditation Council for Business Schools and Programs (ACBSP, 2018), en donde participaron más de 5000 estudiantes inscritos en programas educativos de negocios y economía de 26 universidades de ocho países latinos, se encontró que si bien estas escuelas han tenido éxito relativo para formar emprendedores convencionales, existe un desafío importante en la promoción, desarrollo y consolidación de los emprendimientos sociales, entendido como un modelo de desarrollo socioeconómico que busca superar las fallas del gobierno y del mercado, generando un efecto positivo en un modelo empresarial que aborde desafíos sociales como la pobreza (Koch, 2010).

Los resultados del estudio arrojan que sólo el $3.4 \%$ de los estudiantes han presentado un emprendimiento social, a pesar de que el $62 \%$ ha tenido intenciones de emprender. Respecto a los determinantes principales, resalta tener buena imagen del emprendedor social, contar con el apoyo social y sentirse competente. En este sentido, se concluye que la formación universitaria requiere reenfocar sus estrategias pedagógicas para aumentar la eficacia en el emprendimiento social. Si bien la promoción de estos tipos de emprendimientos es hoy un eslabón fundamental para combatir los problemas 
ligados a la condición de pobreza, también es importante ligar las misiones de las instituciones de educación con los requisitos del emprendimiento social, con la intención de generar un impacto mayor (ACBSP, 2018).

El análisis de ACBSP encuentra que en México y Latinoamérica las instituciones educativas están incluyendo en sus planes de estudio el tema del emprendimiento social, sobre todo en aspectos teóricosconceptuales, por lo que el interés en este enfoque ha crecido de manera exponencial en los últimos años. No obstante, es evidente la ausencia de un eslabón intermedio que lleve al estudiante a consolidar, en los hechos, un proyecto social que transite del conocimiento teórico y técnico, al práctico.

La mayoría de estudios de evaluación de eficiencia universitaria se han basado en medir el impacto de la formación a partir de la intención emprendedora, pero no en la conducta, existiendo una brecha entre la intención y los resultados (o los hechos). En términos generales, se encontró que el emprendedor social tiene mayor intención emprendedora, se siente más capaz, fomenta mayor participación de las mujeres, e inclusive tiene mayor aprobación de su entorno, sin embargo, el nivel de estudiantes que realmente opera o practica un emprendimiento social, es bajo (ACBSP, 2018).

\section{Los emprendimientos de negocios sociales}

Cada vez más se fortalece el sentido de pertinencia y aportación que generan los emprendimientos sociales para combatir el rezago y la pobreza en zonas vulnerables. En el ámbito académico, diversas publicaciones han discutido o resaltado su relevancia. Junto con ello, han surgido o retomado importancia conceptos como economía social, innovación social y específicamente, negocios sociales. Éstos pretenden abordar el estudio de experiencias emprendedoras que influyan en el diseño e implementación de programas y acciones que apunten hacia la consecución de un desarrollo más equilibrado, justo y sustentable, que coadyuve a la erradicación de la pobreza (Yunus, 2013).

Un negocio es social cuando el emprendedor o dueño enfoca sus esfuerzos en resolver un problema social de forma sostenible, en lugar de buscar ganancias monetarias personales o colectivas. Así, para que el negocio sea sostenible, éste debe dirigirse de manera similar a cualquier empresa en un mercado, en el sentido que debe optimizar recursos, evaluando estructuras, políticas y régimen regulatorio, pero vendiendo bienes o servicios a un precio mínimo necesario para solventar costos y recuperar inversión (Khashru, 2017).

La diferencia principal con la empresa convencional es que un negocio social utiliza las posibles ganancias adquiridas para expandir sus operaciones con el objetivo de resolver más problemas sociales. Así, todo negocio existente podría convertirse en negocio social si el dueño o los dueños deciden reconfigurar sus objetivos hacia la solución de problemas sociales, eliminando los fines lucrativos. Un negocio social no es un proyecto de caridad que se sustente en donaciones, aportaciones voluntarias o gubernamentales (Yunus, 2013). Por ello, todo negocio social deriva de un emprendimiento social, pero no todos los emprendimientos sociales o las innovaciones sociales deben ser negocios sociales.

Existen dos tipos de negocios sociales: aquellos que se centran en proporcionar un beneficio social y no en maximizar los beneficios para los propietarios; y aquellos cuyos dueños provienen de zonas o sectores pobres. En este último caso, el beneficio social se deriva del hecho de que las ganancias y el crecimiento de la empresa se destina a la obtención de ingresos para el círculo familiar del emprendedor, lo que ayuda a reducir su pobreza o incluso a escapar de ella (Khashru, 2017).

Bajo esta visión, si se observa el total de unidades económicas en México, las microempresas representan el 95.2\%; las pequeñas el 4.3\%; las medianas el $0.3 \%$ y las grandes el $0.2 \%$. Las microempresas 
también son el sector empresarial más importante en generación de empleo, concentra el $45.6 \%$, las pequeñas al $23.8 \%$ y las medianas $9.1 \%$, y el resto se ocupa en las grandes empresas. En general, se sabe y acepta que el promedio de vida de las microempresas es bajo, el 45\% tienen hasta cinco años de antigüedad. Esto puede explicarse por las condiciones en que operan, pues los tres principales problemas que éstas enfrentan por sus mercados reducidos en México, son bajas ganancias, competencia excesiva y aumento en precios de los insumos y mercancías (INEGI, 2018).

El principal motivo de emprendimiento del más amplio sector microempresarial es complementar el ingreso familiar, conseguir un ingreso mayor en comparación al salario asociado a un trabajo mal pagado, o porque no encontró empleo en alguna empresa (Mungaray et al., 2017). Así, la mayoría de las microempresas son formadas por individuos en situación vulnerable, que ante escenarios de desempleo y/o la falta de ingresos monetarios, emprenden un negocio cuya intención es la obtención de un nivel mínimo de subsistencia para el propietario y su familia, sin la preparación necesaria o suficiente respecto a mercados o negocios. En este sentido, la microempresa se asocia a un negocio social tipo dos.

\section{Antecedentes del proyecto de aprendizaje- servicio en la UABC}

Consciente de este contexto social y empresarial local y nacional, la UABC se ha caracterizado en los últimos 20 años por realizar una labor de vinculación comunitaria y responsabilidad social, a través de la gestión y estructuración de ABP, gracias a iniciativas de académicos y estudiantes, en apoyo a microempresas. Esto comenzó con el Programa de Investigación, Asistencia y Docencia a la Micro y Pequeña Empresa (PIADMyPE), creado en 1999 como un proyecto de servicio social de estudiantes de licenciatura en apoyo a microempresas, con recursos de la Fundación Ford, Organización de los
Estados Americanos (OEA), la Asociación Nacional de Universidades e Instituciones de Educación Superior en México (ANUIES) y el Gobierno Federal Mexicano a través de la Secretaría de Educación Pública (SEP), el Consejo Nacional de Ciencia y Tecnología (CONACyT) y el Fondo Nacional de Empresas Sociales. El propósito principal de esta iniciativa fue contribuir al desarrollo de microempresas de base social y bajo valor agregado, con el apoyo de estudiantes universitarios en servicio social a través de su asistencia y entrenamiento. El PIADMyPE inició con los siguientes objetivos:

- Construir un modelo de enseñanza y aprendizaje en servicio para estudiantes de economía y áreas afines.

- Brindar servicios de desarrollo empresarial in situ y sin costo, a microempresas sociales ubicadas en las colonias con mayor índice de pobreza.

- Realizar investigación en temas relacionados con el desarrollo de empresas de base social, a través del modelo de investigación-acción para el fortalecimiento curricular de programas de licenciaturas en economía y áreas afines.

En 2006, inspirados en el concepto de investigación-acción del PIADMyPE y con el objetivo de fortalecer la vinculación con este sector microempresarial de la región, de manera más institucional, el programa se formaliza en Centro de Investigación, Asistencia y Docencia de la Micro y Pequeña Empresa (CIADMyPE). De esta forma, en 2009, en el contexto de la gran crisis mundial de fines de 2008, se promueve el vínculo con el gobierno del estado de Baja California a través de la Secretaría de Desarrollo Económico, y el Gobierno Federal con la política de apoyo empresarial llamado Fondo PyME, para utilizar el CIADMyPE como elemento base de una política pública contracíclica, con intención de hacer frente a los impactos de inestabilidad y desempleo 
que trajo consigo la crisis económica, ante el despido de miles de trabajadores de las empresas medianas y grandes, principalmente en las llamadas maquiladoras, que buscando mantener su competitividad global, se desentendieron de sus compromisos laborales y sociales.

Con esta evolución, se establecieron los siguientes objetivos:

- Con base en la participación universitaria, a través de docentes y estudiantes, construir una política pública para el desarrollo microempresarial de base social y fomentar el empleo, a través de la retroalimentación y experiencia adquirida en el Proyecto PIADMyPE.

- Generar programas de Aprendizaje Basado en Proyectos alternos para poner en práctica competencias adquiridas de los estudiantes en sus programas educativos, para la formalización y vinculación de empresas sociales en ventanillas de financiamiento. Esto se institucionalizó en la UABC a través de figura de Proyectos de Vinculación con valor en créditos curriculares.

Con una visión global de innovación social, en 2017 el CIADMyPE se transforma en UABC-Centro Yunus para los negocios sociales y el bienestar, con el fin de profundizar la vocación de responsabilidad social en sus estudiantes, mediante el desarrollo de capacidades de intervención comunitaria, orientadas a promover y organizar a los negocios sociales, para resolver problemas de la sociedad en sus segmentos más vulnerables, bajo las ideas y filosofía del profesor $\mathrm{Mu}-$ hammad Yunus, premio nobel de la paz 2006. Los objetivos principales de UABC-Centro Yunus, son:

- Desarrollar modelos de aprendizaje-servicio innovadores que permitan la transmisión y adquisición de competencias técnicas y solidarias entre los estudiantes universitarios, negocios sociales y profesionistas.
- Combatir a la condición de pobreza y desigualdad a través de la creación y consolidación de negocios sociales y generar redes solidarias de colaboración local, regional, nacional e internacional entre academia, gobierno y sector privado.

Durante la evolución de este programa, un total de 1722 estudiantes de licenciatura han participado en las actividades operativas que conducen el desarrollo y asistencia a negocios sociales, efectuando su servicio social profesional y prácticas profesionales, fortaleciendo de esta manera su aprendizaje formativo, su sentido solidario y orientando su conducta de emprendedor social, facilitando el tránsito del conocimiento a la práctica.

\section{Metodología del programa de aprendizaje- servicio: asistencia y enseñanza a negocios sociales}

El programa de Asistencia y enseñanza a negocios sociales brinda a los estudiantes la oportunidad de ejercer su profesión, acceder a su contexto social y desarrollar el sentimiento de afecto por su comunidad y el valor de la solidaridad, a través de los emprendimientos sociales, especialmente, los negocios sociales (Mungaray et al., 2020). Actualmente, los estudiantes universitarios de la UABC, tanto de la Facultad de Economía y Relaciones Internacionales, en la ciudad de Tijuana, como de las facultades de Ingeniería y Negocios en las poblaciones semiurbanas y rurales de Guadalupe Victoria, Tecate y San Quintín, en Baja California, asisten a microempresas sociales (negocios sociales tipo dos) durante un periodo de 12 semanas, trabajando como consultores, desarrollando un programa completo de recolección, procesamiento y análisis de datos, que termina en un informe sobre el desempeño de la empresa y plan de mejora y seguimiento que se proporciona a los microempresarios sociales.

Durante este proceso, los estudiantes y profesores se involucran con los dueños de los negocios, 
transfieren conocimientos, conceptos económicos, empresariales y habilidades técnicas y de gestión, lo que permite a este sector empresarial adquirir un capital humano que de otro modo sería imposible. El dinamismo de este programa también permite que los empresarios sociales transfieran a los estudiantes la experiencia adquirida de emprender un negocio en contextos vulnerables y de limitaciones importantes en recursos, tanto tangibles como intangibles. La asistencia in situ constituye una respuesta inmediata a los problemas específicos que enfrenta la región en condiciones de marginación y pobreza, además de una experiencia de vida diaria para los estudiantes universitarios.

La demostración de que el programa ha tenido la capacidad de desarrollar modelos de enseñanza y aprendizaje innovadores para los alumnos en servicio, requiere mostrar el proceso mediante el cual ocurre la transmisión y adquisición de competencias técnicas y solidarias por parte de los microempresarios. Para ello se detallan las cinco partes en que el programa está estructurado: reclutamiento y entrenamiento a estudiantes, selección de negocios sociales, asistencia individual y en grupo a negocios sociales, análisis empresarial y acompañamiento.

\section{Reclutamiento y entrenamiento a estudiantes}

El programa se basa principalmente en estudiantes de la licenciatura en Economía, pero también incorpora a estudiantes de campos afines, por ejemplo, de gestión, contabilidad, negocios, administración pública y marketing, que presenten un interés genuino en apoyar a negocios sociales. Para ello, previamente se publica una convocatoria invitando a alumnos en etapa terminal de licenciatura, a incorporarse al programa a través de prestación de servicio social y prácticas profesionales. Los profesores-coordinadores del programa evalúan las solicitudes de inscripción de cada estudiante, según su perfil e intereses. Una vez seleccionados, se hacen reuniones destinadas a familiarizar a los alumnos y describir los componentes principales del programa, además de enfatizar la importancia de trabajo con sectores microempresariales de zonas marginadas, así como la relevancia de su participación en el programa.

El programa ofrece un modelo de capacitación y entrenamiento para los estudiantes participantes. Éste es destinado a cubrir algunos de los elementos básicos de desarrollo empresarial, que los alumnos aplican en los negocios sociales, con el fin de estandarizar en ellos el marco de conceptos. A todos los participantes se les comparten temas asociados a emprendimiento social, innovación social y desarrollo comunitario. Además, se les imparte un entrenamiento específico, según su formación, con el cual direccionan la asistencia a las microempresas, con base en las siguientes clasificaciones:

- Estudiante de Economía, Contabilidad, Administración y afines: capacitación central al estudiante en temas de administración, compras, ventas y finanzas, para microempresarios.

- Estudiante de Administración pública y Ciencia políti$c a$ : capacitación central al estudiante en temas de gestión social (gubernamental), acceso a créditos y formalización para microempresarios.

- Estudiante de Relaciones internacionales y afines: capacitación central al estudiante en temas de cooperación y creación de redes para la vinculación con proyectos o fondos locales, nacionales o internacionales, para microempresarios.

- Estudiante de Psicología: capacitación central al estudiante en temas de empoderamiento, liderazgo personal y desarrollo comunitario.

La experiencia estudiantil en la operación del programa juega un papel importante en la provisión de casos reales y contextos complicados para el éxito empresarial, por lo que exige la aplicación de competencias técnicas y sociales, aprendidas por los estudiantes durante sus carreras, usando como 
referencia escenarios y datos de microempresarios atendidos previamente.

\section{Selección de negocios sociales}

Una de las desventajas de algunos programas de apoyo empresarial es que los empresarios deben asistir a las ubicaciones del proveedor de la capacitación, y no al revés. Esto se convierte en un importante elemento que desalienta a los dueños de negocios a recibir servicios de asistencia, ya que tienen que abandonar sus tareas y rutinas de la empresa. Dado que ellos, la mayoría de las veces, son los únicos que laboran en su negocio, desarrollan múltiples tareas como gestión, producción, ventas, entre otras, por lo que dejar su empresa para ir a recibir la capacitación, produce un costo de oportunidad considerable.

Para superar esto, este programa considera en su estructura la asistencia in situ, para lo cual los propios alumnos buscan a los negocios sociales por diversas maneras, ya sea visitando barrios marginales de puerta en puerta, visitando a centros comunitarios, colegios o centros de asistencia de los barrios. Otros mecanismos de contacto son las oficinas del gobierno local y las propias instalaciones universitarias.

Los microempresarios sociales operan dentro de sus hogares, en una figura de talleres familiares o independientes. Otras microempresas son más visibles, al utilizar puestos semifijos o instalaciones externas a la estructura de su hogar. Las microempresas elegibles para el programa son aquellas que se consideran desfavorecidas social y económicamente, reflejado, por ejemplo, en la producción de bienes con bajo valor agregado, que operan mayoritariamente en el sector informal, cuyo único propietario realiza muchas de las actividades relevantes en el negocio, empleando principalmente a miembros de la familia, sin separación entre los recursos del negocio y el hogar.

Si bien en México se considera como microempresa a aquellas unidades que no tienen más de 10 empleados, los negocios sociales atendidos por este programa tienen entre dos y cinco trabajadores máximo, donde la familia juega un papel importante. Esta definición es un criterio práctico de elegibilidad por parte de los estudiantes cuando están realizando la búsqueda de las microempresas que asistirán. Además, las empresas no deben contar con el apoyo oficial o institucional de algún otro programa, debido a su falta de registro formal ante la autoridad fiscal o la falta de registros técnicos, contables y administrativos. Adicionalmente, la mayoría de los propietarios deberían tener educación primaria, secundaria o preparatoria. Estos criterios facilitan el acceso a los negocios sociales más modestos y aseguran la relevancia del trabajo realizado por los estudiantes, además de que dan cuenta de la rentabilidad social del proyecto.

\section{Asistencia individual y en grupo}

El trabajo de apoyar a los negocios sociales requiere varias visitas y entrevistas con el empresario de manera individual e in situ, es decir, en las mismas instalaciones del negocio social. Algunas capacitaciones se realizan en forma grupal y se llevan a cabo en los mismos barrios donde se ubiquen. Durante los tres meses de asistencia, se proporciona un marco de instrumentos y herramientas que formalizan la relación entre los alumnos y empresarios, acompañados por docentes. El programa de capacitación se conforma por cinco instrumentos de aplicación: Diagnóstico, Manual de fortalecimiento, Bitácoras, Entregables de capacitación, Valoración-evaluación.

- El diagnóstico se aplica al inicio de la implementación de la asistencia mediante una codificación de criterios y gráfico de redes, además de una reseña histórica de los motivos del emprendimiento. El estudiante debe identificar las áreas de oportunidad, tanto personales como empresariales del dueño del negocio, los cuales se analizan con detalle dentro de la asistencia.

- El manual de fortalecimiento tiene la intención de servir de guía y sustento para los estudiantes 
que llevan a cabo la labor de consultores. Se desarrollan temas de desarrollo empresarial, además de apartados especializados según el área de cada estudiante, como gestión gubernamental, finanzas, empoderamiento personal, vinculación a redes de colaboración y desarrollo comunitario. Cabe destacar que este manual se modifica a partir de la retroalimentación y aprendizaje recibido de aplicaciones anteriores, a través de un trabajo colegiado entre docentes y estudiantes, en el marco de la asignatura de "Economía de la pequeña y mediana empresa", impartida en el programa de licenciatura en Economía. En función de los puntos débiles que se identifican en la aplicación del diagnóstico, y el estudio del manual de fortalecimiento, el estudiante, junto con los profesores-coordinadores del programa, realizan un plan personalizado de atención al negocio social. El manual de fortalecimiento garantiza que la capacitación otorgue una serie de conceptos básicos que el estudiante debe transmitir al empresario, con el fin de mejorar la operación de su negocio.

- Las bitácoras son una herramienta para el registro de entradas y salidas de producto y/o dinero, clasificando los tipos de gastos e ingresos para el cálculo de saldos diarios y la administración y asignación eficiente de recursos.

- En los entregables de capacitación empresarial se compilan los productos y mejorías que se realizan al negocio social respecto de los temas que aborda la asistencia. Tiene la intención de sintetizar, presentar y entregar de forma tangible al empresario el trabajo que los estudiantes logran para el desarrollo y solidificación del negocio social.

- El formato de valoración-evaluación tiene una función dual: por un lado, apreciar de una forma tangible, el avance del empresario en sus actividades microempresariales, al valorar tanto su perfil de ingreso como de egreso a la asistencia, aplicando los mismos criterios al inicio y al final de las actividades de capacitación, efectuando una evaluación de la eficiencia del proyecto de asistencia, respecto a los objetivos e intenciones que se establecieron al comienzo de la capacitación, tanto para el dueño de la empresa como para el estudiante. Por otro lado, el estudiante se somete a evaluación realizada por el profesor-coordinador del proyecto, en conjunto con el dueño del negocio asistido, en donde se le preguntan aspectos de desempeño, comunicación, conocimiento técnico, valores, conducta, responsabilidad, disciplina y puntualidad del estudiante. Con dicha información, el alumno recibe una retroalimentación de su trabajo durante el periodo de la asistencia. Además, el estudiante presenta un documento-informe, en donde describe de manera estructurada cada una de las actividades y resultados alcanzados. Este documento es evaluado por un profesor externo al programa, invitado para ser lector de dicho informe. Todos los alumnos participantes comparten sus experiencias en un coloquio semestral, en donde el resto de estudiantes de licenciatura y docentes tienen la oportunidad de conocer las experiencias y resultados obtenidos durante la operación de este programa.

\section{Análisis empresarial para posible financiamiento}

Al final de los tres meses de asistencia, los estudiantes analizan los datos que recopilaron con el fin de evaluar formalmente la viabilidad de un financiamiento para el empresario. Esta etapa se desarrolla con el soporte del software con derechos de autor: "Sistema para la Asistencia Microempresaria" (SAM), el cual genera informes económicos y financieros como análisis de costos, estado de resultados, flujos de efectivo, declaración de ingresos y análisis de razones financieras, de todas las microempresas atendidas. 
El software permite registrar y almacenar información socioeconómica de los negocios sociales que participan en el programa, como datos personales, educación, experiencia, capital, activos, estructura financiera, estrategias, ingresos, costos y gastos, con los cuales se realizan estudios académicos y de investigación aplicada, al permitir generar de manera sistemática una base de datos a partir de fuentes primarias. Gracias a ello se ha consolidado la realización de actividades paralelas con estudiantes que colaboran en la realización de proyectos de investigación como tesis, libros, capítulos de libros, artículos y ponencias, siempre con celoso resguardo y confidencialidad de datos personales.

Con el análisis empresarial se canaliza al negocio social a ventanillas disponibles de financiamiento, en donde sea posible conseguir algún apoyo monetario para la operación de su negocio, ya sea en forma de crédito o subsidio. En diversos periodos, el programa se ha vinculado con dependencias gubernamentales o privadas, las cuales han apoyado con los recursos para el financiamiento, previa capacitación y determinación de los montos óptimos para cada negocio social. Sin embargo, si bien la formalización y veracidad del plan de negocio presentado a la institución de fondeo es considerado para la evaluación académica del estudiante, la adquisición el financiamiento no es considerado dentro de la evaluación del alumno, debido a que esto obedece principalmente a los objetivos y decisiones de los entes externos de fondeo, los cuales, muchas de las veces, consideran aspectos ajenos a la propia capacitación.

\section{Acompañamiento}

Una vez que los microempresarios aceptaron el desafío de estar en una capacitación continua de tres meses, operar su negocio en un contexto de formalidad y la probabilidad de recibir un apoyo financiero, el programa continúa en una etapa adicional de acompañamiento a distancia, con ello se busca apoyar el desempeño del micronegocio social ante los nuevos escenarios de operación, así como en las nuevas obligaciones que se tienen ante la autoridad tributaria o financiera. Este seguimiento se desarrolla a través de dos talleres grupales sobre temas de finanzas, marketing y seguridad en el negocio; éstos están destinados al empresario para aprender sobre cómo solicitar financiación ante la banca comercial, requisitos financieros, implicaciones del apalancamiento empresarial, monto de financiamiento, tasa de interés, periodo de gracia, el concepto de riesgo, costo de oportunidad, inventario a corto y largo plazo, inversión en maquinaria y equipo, ventas, ingresos y salarios. Estas actividades continúan realizándose principalmente, con apoyo de egresados del área de negocios y economía.

\section{Resultados}

Hasta 2019, el programa incorporó a 1722 estudiantes que trabajaron como consultores, viviendo y aprendiendo de la experiencia de una empresa en contextos de alta vulnerabilidad, y consolidando sus conocimientos técnicos y solidarios vistos en clases, visualizando a los negocios sociales como un mecanismo viable y sostenible para combatir la pobreza. De esta forma, se ha propiciado un cambio sustancial en la relación educativa, modificando el énfasis de la enseñanza y aprendizaje, a través de métodos y prácticas relacionados con la investigación-acción y aprendizaje-servicio, principalmente. La evidencia respecto a la utilidad de los consejos de los estudiantes para los negocios sociales, puede considerarse como una forma muy exigente de medir su aprendizaje universitario.

De los estudiantes que han participado en el programa de asistencia a negocios sociales, el $60 \%$ ha expresado que las experiencias adquiridas les han ayudado principalmente en su desarrollo personal, mientras que el resto pondera en mayor medida el impacto en su desempeño laboral. El 40\% considera que el programa de asistencia a negocios sociales le brindó la experiencia necesaria para continuar 
desempeñándose en el medio, para laborar o cooperar dentro de un negocio social; mientras que el 15\% ha realizado estudios de actualización en temas de emprendimiento social e innovación social. El 60\% estaría interesado en seguir especializándose en estudios de posgrado, pues el $70 \%$ de éstos considera necesario tener un conocimiento técnico para realizar proyectos que impacten y brinden soluciones viables a problemas de la sociedad ligados a la pobreza y desigualdad.

El 25\% de los estudiantes resaltan la estructura innovadora de enseñanza y aprendizaje del programa, otro $25 \%$ resalta las alianzas con dependencias y organismos locales, nacionales o internacionales, y el 50\% que sea un programa de asistencia in situ. En temas de retroalimentación curricular, los estudiantes participantes consideran que los cuatros temas principales que todos los programas académicos de ciencias sociales deben considerar son: metodologías de intervención y evaluación de impacto, gestión de recursos, vinculación y creación de redes comunitarias y habilidades para la elaboración de proyectos sociales.

El impacto académico, educativo y social del programa ha sido atractivo para organizaciones educativas, gubernamentales y no gubernamentales, que en periodos específicos han financiado su operación con el objetivo de transformar dicho programa en un modelo que permita reproducirlo a escala nacional o internacional. Así, una vez iniciado en Baja California, con los fondos proporcionados por la
Fundación Ford, el CONACyT y la OEA, fue posible operar a mayor escala y de manera sistemática a través de distintas instituciones de educación superior.

Del periodo 2001-2007, el número de estudiantes y negocios sociales apoyados aumentó al implementar el programa en ciudades de los estados mexicanos de Baja California Sur, Sonora, Sinaloa, Ciudad de México, Nayarit y Yucatán. En esos años, en total se asistió a 1573 negocios sociales con la participación de 98 estudiantes.

De 2009 a 2013, el gobierno del estado de Baja California, a través de la Secretaria de Desarrollo Económico y la UABC, implementaron un programa integral de asistencia a microempresas sociales, convirtiéndose en una política pública local para enfrentar el desempleo generado por el despido de miles de trabajadores por parte de la industria maquiladora y manufacturera de exportación, ante la caída de la demanda mundial y la inclusión de tecnologías de producción modernas para economizar mano de obra y aumentar su productividad. En este periodo se apoyó a más de 10000 negocios sociales con la participación de más de 1300 alumnos.

A partir de 2017, el programa se adhirió a la Red Global de los Centros Yunus, con la creación de UABC-Centro Yunus, de la Facultad de Economía y Relaciones Internacionales de la UABC, con el cual se empieza a construir la colaboración con los cerca de 50 Centros Yunus instalados en universidades alrededor del mundo (ver tabla 1).

Tabla 1. Negocios sociales y estudiantes en el programa de asistencia empresarial, 1999-2019

\begin{tabular}{l|c|c|c|c|c|c|c|c}
\hline Concepto & $\mathbf{1 9 9 9 - 2 0 0 8}$ & $\mathbf{2 0 0 9 - 2 0 1 3}$ & $\mathbf{2 0 1 4}$ & $\mathbf{2 0 1 5}$ & $\mathbf{2 0 1 6}$ & $\mathbf{2 0 1 7}$ & $\mathbf{2 0 1 8}$ & \\
\hline $\begin{array}{l}\text { Negocios } \\
\text { apoyados }\end{array}$ & 1015 & 10047 & 619 & 424 & 218 & 118 & 200 \\
\hline $\begin{array}{l}\text { Estudiantes } \\
\text { participantes }\end{array}$ & 98 & 1302 & 77 & 62 & 43 & 40 & 55 \\
\hline
\end{tabular}

Fuente: elaboración propia 
En este sentido, el programa también ha reflejado su responsabilidad social y comunitaria al apoyar a más de 12790 negocios sociales de áreas urbanas y rurales. Esta cantidad de empresas recibió asistencia y, la mayoría, un apoyo financiero. La mayoría también fueron incorporados al mundo formal, promoviendo el registro ante la autoridad fiscal bajo el régimen del pequeño contribuyente. El apoyo financiero no reembolsable alcanza una bolsa de $28 \mathrm{mi}-$ llones de pesos, proporcionados a través de gestiones ante dependencias públicas y organizaciones no gubernamentales, nacionales e internacionales.

De los negocios sociales atendidos, $39 \%$ son talleres familiares, $35 \%$ talleres independientes, $14 \%$ puestos semifijos en la vía pública y el resto son negocios ambulantes. Respecto a la educación de los empresarios, $33 \%$ tienen hasta nivel de primaria, 21\% secundaria, $22 \%$ preparatoria y el resto no tienen estudios. La edad de los dueños del negocio oscila entre los 35 y 60 años, al momento de recibir la capacitación por parte de los estudiantes. Es importante tener en cuenta que el programa ha beneficiado principalmente al sector femenino, sin ser un objetivo a priori, ya que el $73 \%$ de los empresarios apoyados son mujeres, esto quizá puede reflejar una proporción natural de las características imperantes de este sector empresarial.

Una externalidad positiva observada del programa, es que a partir de los casos de éxito de las microempresas atendidas, algunos de los familiares y vecinos de los dueños participantes han mostrado intenciones de consolidar su propio proyecto de emprendimiento social, por lo que en 2019 se estructuró un proyecto piloto para operar un programa de emprendimiento social, por parte de los estudiantes, en una figura de incubadora para que el emprendedor realice un plan de negocio social viable, con impacto familiar o comunitario en zonas de atención prioritarias, y con posibilidad de acceso a algún apoyo financiero, público o privado.

En este sentido, los estudiantes llevaron una asistencia individualizada con cada emprendedor, en donde el alumno de Economía le asiste en la creación del plan de negocio para analizar la viabilidad de la idea, costo y mercado del posible producto o servicio a ofertar; el estudiante de Administración Pública y Ciencia Política le apoya en el plan de gestión de recursos para la financiación del plan de negocio; mientras que el alumno especializado en el área de Psicología y Trabajo Social implementó todo un plan de desarrollo de liderazgo. En total se atendió a 28 emprendedores en este programa piloto, divididos en cuatro grupos, en donde cada estudiante apoyaba de manera individual a cada emprendedor social con dos sesiones por semana. Las ideas de negocios que se diseñaron fueron principalmente en el sector de servicios y comercio. La edad promedio de los emprendedores fue de 40 años, 16 de ellos eran mujeres. Como parte de los resultados de esta prueba piloto, se evidenció un desempeño positivo en la adquisición y transmisión de habilidades, tanto en el estudiante como en el emprendedor, por lo que en el futuro se estudiará cómo sistematizar esta actividad como parte complementaria del proyecto de aprendizajeservicio de asistencia a negocios sociales.

Gracias a los instrumentos que recopilan información sumativa del proyecto de asistencia a negocios sociales, es posible comprobar que este prorgama brinda valioso apoyo pedagógico a los estudiantes para adquirir experiencias por parte de los dueños de negocios sociales, en la creación y operación de una empresa, muchas de las veces, en las circunstancias más adversas, demostrando un genuino espíritu de superación, en donde los alumnos se ven altamente influenciados por el comportamiento y ejemplo que proporciona este sector poblacional y emprendedor. Incluso, se ha evidenciado que algunos estudiantes desarrollan con los dueños de las empresas una relación de amistad y colaboración, que perdura más allá del periodo institucional del proyecto.

En suma, 56\% de los estudiantes participantes realizan o han realizado una actividad emprendedora. Cerca del 80\% de los alumnos manifestaron 
haber desarrollado una mayor inspiración, motivación y confianza, una vez realizado el proceso de asistencia a los negocios sociales.

Finalmente, el software de recopilación de información microempresarial, SAM, ha fungido como proveedor de datos originales y reales del contexto empresarial local, para las asignaturas de licenciatura como ingeniería económica, formulación de proyectos, evaluación de proyectos, economía de la pequeña empresa, microeconomía intermedia, entre otros; pero además es la base de estudios e investigaciones reflejados en productos académicos como libros, capítulos de libros y artículos con arbitraje nacional e internacional, en donde se han resaltado y discutido los temas teóricos-conceptuales del emprendimiento social, emprendimiento por necesidad, pull-push-efect, objetivos económicos de la microempresa, competitividad microempresarial, políticas públicas de apoyo microempresarial, microfinanciamiento, etcétera, en donde los estudiantes han tenido la oportunidad de colaborar en actividades de investigación aplicada.

\section{Conclusiones}

La colaboración estudiantil con los microempresarios pobres ha generado importantes beneficios sociales para los estudiantes participantes. Por un lado, permite evaluar su desempeño y conducta ante escenarios reales de negocios sociales, lo cual adiciona un elemento para poner a prueba su comportamiento profesional ante contextos de marginación alta, viviendo directamente la experiencia de un emprendimiento social. Por otro lado, permite evaluar el aprendizaje adquirido por el estudiante en el salón de clases, a través de la evidencia observada en la mejora de los indicadores de desarrollo del negocio, existiendo espacios para la retroalimentación y acompañamiento por parte de docentes, tanto para el estudiante como para el microempresario social. Esto permite evaluar la eficiencia de cada asistencia estudiantil, a través de los resultados obtenidos en cada micronegocio social atendido.

Por otra parte, los resultados de la aplicación de este programa para el desarrollo de negocios sociales, sugieren la posibilidad de brindar capacitación efectiva, asistencia para la formalización, alternativas para el combate a la condición de pobreza y consolidación de responsabilidad social, a partir de una metodología pedagógica y universitaria de investigación-acción, con la participación central de estudiantes de licenciatura. También ha demostrado que los esquemas de colaboración, en este caso con el gobierno, las universidades, organismos no gubernamentales y los negocios sociales, pueden resultar en políticas públicas efectivas.

Las distintas evaluaciones de este programa ilustran que no sólo se ha capacitado a más de 12790 negocios sociales, sino que también se muestra la posibilidad de que una selección de estas empresas pueda aspirar a niveles más altos de desarrollo, a partir de asistencias personalizadas por estudiantes universitarios, apoyos financieros y consultorías in situ. Ésta es una piedra angular en la mejora del bienestar de los dueños de los negocios, personas que viven en su entorno, y también en la formación técnica y solidaria de los jóvenes estudiantes universitarios.

Hacia el futuro, el desafío es que todas las empresas sociales de este tipo puedan perdurar en el tiempo y ser más autosuficientes. Esto permitirá que el programa continué operando sustentablemente para los microempresarios. Esta experiencia por parte de los estudiantes ilustra lo que es posible lograr socialmente con iniciativas emprendedoras, sustentables y trabajo colaborativo para atender la pobreza. 


\section{Referencias}

Accreditation Council for Business Schools and Programs (ACBSP) (2018), El emprendimiento social de base universitaria en Latinoamérica, Lima, ACBSP/Universidad de San Martín de Porres.

Barba-Sánchez, Virginia y Carlos Atienza-Sahuquillo (2016), "The development of entrepreneurship at school: the Spanish experience", Education and Training, vol. 58, núm. 7-8, pp. 783-796.

Barbero, Josep Manuel y Ferrán Cortés (2005), Trabajo comunitario, organización y desarrollo social, Madrid, Alianza Editorial.

Bausela-Herrera, E. (2004), "La docencia a través de la investigación-acción", Revista Iberoamericana de Educación, vol. 35, núm. 1, pp. 1-9.

Botella, Ana María y Pablo Ramos Ramos (2019), "Investigación-acción y aprendizaje basado en proyectos. Una revisión bibliográfica”, Perfiles Educativos, vol. 41, núm. 163.

Cabrera Morgan, L. (2017), "La investigación-acción: una propuesta para la formación y titulación en las carreras de Educación inicial y primaria de una institución de educación superior privada de Lima", Educación, núm. 51, pp. 137-157.

Consejo Nacional de Evaluación de la Política de Desarrollo Social (CONEVAL) (2017), "Medición de la pobreza, pobreza en México", <https://www.coneval. org.mx/Medicion/Paginas/PobrezaInicio.aspx > [Consulta: noviembre de 2019].

Consejo Nacional de Población (CONAPO) (2017), Datos de proyecciones por entidad 2016-2020 y Conciliación demográfica de México, 1950 -2015, Ciudad de México, CONAPO-SEGOB.

Chiva, Bartoll, Capella Peris y Pallarès Piquer (2018), "Investigación-acción sobre un programa de aprendizajeservicio en la didáctica de la educación física" Revista de Investigación Educativa, vol. 36, núm. 1, pp. 277-293, DOI: http://dx.doi.org/10.6018/rie.36.1.270581.

García, Angela, Aurelio Villa y Aitziber Mugarra (2018), "Procesos de investigación-acción en aprendizaje y servicio solidario: percepciones de los protagonistas pedagógicos en el proyecto Hiri Lagunkoiak", Revista Iberoamericana de Aprendizaje Servicio, vol. 5, pp. 62-86.

González, Juan, Germán Osorio y Alejandro Mungaray (2018), "La microempresa mexicana, un asunto de necesidad y no de oportunidad: el caso de Colima", Revista Análisis Económico, vol. 33, núm. 84, pp. 123-142.

Instituto Nacional de Estadística y Geografía (INEGI) (2018), "Encuesta Nacional sobre Productividad y Competitividad de la Micro, Pequeña y Mediana Empresa (ENAPROCE), 2018", <https://www.inegi. org.mx/programas/enaproce/2018/>, [Consulta: diciembre de 2019].

Kantis, Hugo, Pablo Angelelliy y Moori Virginia (2004), Desarrollo emprendedor: América Latina y la experiencia internacional, Washington D.C., Banco Interamericano de Desarrollo/FUNDES Internacional.

Khashru, Amir (2017), Exploring social business, Dhaka, Ananya editorial.

Koch, James (2010), Social entrepreneurship as a bottom-up model of socioeconomic development, Washington D.C., World Bank Institute.

Mendia, Rafael (2016), "El aprendizaje-servicio: una metodología para la innovación educativa", Revista Convives, vol. 16.

Mungaray, Alejandro, Germán Osorio y Natanael Ramírez (2017), Ensayos económicos sobre microempresas de subsistencia en México, Ciudad de México, MA Porrúa/UABC.

Mungaray, Alejandro, Germán Osorio y Natanael Ramírez (2020), "Explaining social enterprise: toward a theoretical model", Journal of Developmental Entreperneurship, vol. 24, núm. 1, pp. 1-10.

Organización para la Cooperación y el Desarrollo Económicos (OCDE) (2017), Perspectivas económicas de América Latina: juventud, competencias y emprendimiento, Santiago de Chile, OCDE-CAF-CEPAL.

Pérez-Van-Leenden, M. (2019), "La investigación acción en la práctica docente. Un análisis bibliométrico", Revista Internacional de Investigación en Educación, vol. 12, núm.24, 
DOI: https://doi.org/10.11144/Javeriana.m12-24.ncev

Programa de las Naciones Unidas para el Desarrollo (PNUD) (2016), Informe sobre desarrollo humano, desigualdad $y$ movilidad: México 2016, Ciudad de México, PNUD.

Premchander, Smita (2003), "NGOs and local MFIs, how to increase poverty reduction through women's small and micro-enterprise”, Futures, vol. 35, núm. 4, pp. 361-378.

Ramírez, Natanael, Alejandro Mungaray, José Gabriel Aguilar y Yadira Flores (2017), "Microentrepreneurship as poverty fighting tool: a social evaluation of the mexican case", Revista Innovar, vol. 27, núm. 64, pp. 63-74.

Rubio-Serrano, L. y A. Escofet-Roing (2018), Aprendizajeservicio: claves para su desarrollo en la universidad, Barcelona, Ediciones Octaedro.

Ruiz-Corbella, M.y J. García-Gutiérrez (2019), Aprendizajeservicio: los retos de la evaluación, Madrid, Narcea Ediciones.

Yunus, Muhammad (2013), Creating a world without poverty. Social business and the future of capitalism, Dhaka, Subarna.

\section{Cómo citar este artículo:}

Osorio-Novela, Germán, Alejandro Mungaray-Lagarda y Natanael Ramírez-Angulo (2022), "La colaboración entre estudiantes universitarios y negocios sociales", Revista Iberoamericana de Educación Superior (RIES), vol. XIII, núm. 36, pp. 2643, DOI: https://doi.org/10.22201/iisue.20072872e.2022.36.1182 [Consulta: fecha de última consulta]. 